\title{
SHR, Rat Strain
}

National Cancer Institute

\section{Source}

National Cancer Institute. SHR, Rat Strain. NCI Thesaurus. Code C14412.

SHR: rats develop hypertension spontaneously without exception at the age of 7-15 weeks. There is a systolic blood pressure plateau of about $200 \mathrm{mmHg}$. The genetic basis is polyg enic, with at least three major genes involved (T anase and Suzuki 1971, Yen et al 1974). There is a high incidence of cardiovascular disease (Okamoto et al 1973), but a low incidence of stroke which can be increased to about 30\% with chronic stress (Yamori 1984). 